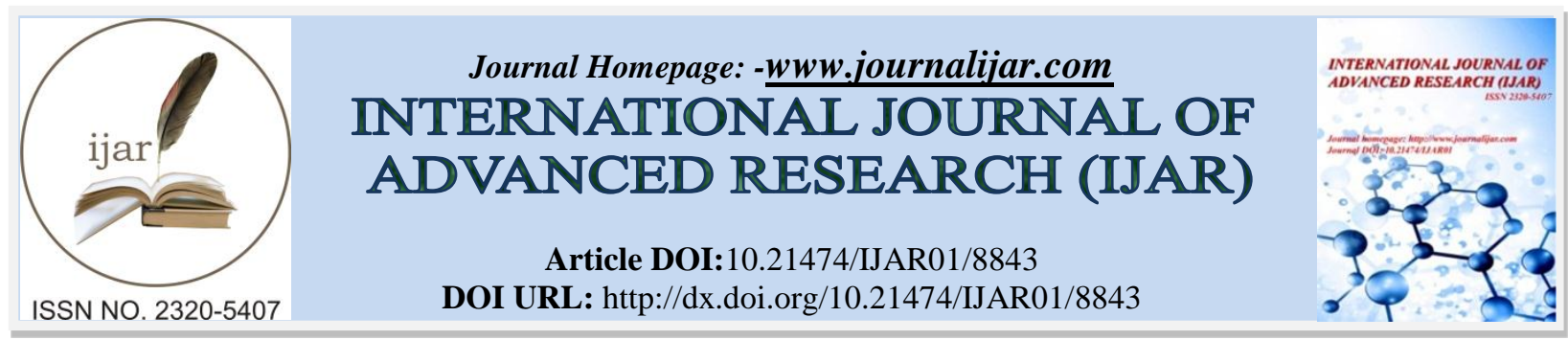

RESEARCH ARTICLE

\title{
DESIGN AND DEVELOPMENT OF NEAR-INFRARED SPECTROSCOPY SYSTEM FOR MONITORING FUNCTIONAL BRAIN ACTIVITY.
}

Nadr Saleh Faleh Alenzi, K. Prahlad Rao and Naif D. Alotaibi.

Department of Electrical and Computer Engineering, Faculty of Engineering, King Abdulaziz University, Jeddah, Saudi Arabia.

\section{Manuscript Info}

Manuscript History

Received: 07 February 2019

Final Accepted: 09 March 2019

Published: April 2019

Key words:-

NIRS, brain activity, Arduino, LED,

Photodiode, Phantom.

\section{Abstract}

Near infrared spectroscopy is an optical technique which can measure the spectral absorbing molecules. Recently, it has been widely researched about its applications because the method is noninvasive and safe. The technology can measure the changes in hemoglobin concentrations in the brain. NIRS is emerging as a promising tool in the cognitive neurosciences, neuro-pathology and psychological studies. Several models of NIRS instruments have been developed, but most of them are either expensive or have safety concerns when laser sources are used. We present a simplified NIRS system using continuous, bicolor LEDs emitting light at $760 / 840 \mathrm{~nm}$, a photodiode with on-chip transimpedance amplifier having highest responsivity for the selected wavelengths. The system is controlled by two Arduino boards, each one for light sources and photodiodes. Since Arduino microcontroller boards are interfaced with the computer, analog to digital conversion (ADC) cards are not necessary in the NIRS system. A sensor probe was designed accommodating the light sources and photodectors. In the probe, LEDs and photodiodes were arranged such that a gap of $3 \mathrm{~cm}$ was maintained between the LED and photodiode in order to monitor the physiological activities in the cortex by measuring the hemodynamic responses. This system has been evaluated by phantom experiment. Observations from experiments suggest that this system can be used for monitoring functional activity of the human brain.

Copy Right, IJAR, 2019,. All rights reserved.

\section{Introduction:-}

Near infrared spectroscopy (NIRS) is an optical technique that can measure physiological parameters such as blood volume and changes in tissue oxygenation (Vaithianathan, et al, 2004) (Kim, et al., 2011). The diagnostic potential of this optical method has been widely explored ever since Jobsis (Jobsis, 1977) demonstrated that the NIR radiation could penetrate deeper into the tissue and the transmittance measurements could be used to monitor the tissue oxygenation. This led to the development of clinical NIRS for monitoring of cerebral function which is safe and non-invasive unlike other methods which uses radio-isotopes of contrast agents (Cope and Delpy, 1988). The technique has great potential in the field of neuroscience (Paol, et al., 2018). When the NIR light in the range of 650$1100 \mathrm{~nm}$ wavelength is incident on the scalp of the head, it penetrates deeper into the brain through the skull and cerebrospinal fluid (CSF). Optical photons from the light source follow different paths within the head. Some of the 
photons get absorbed by the tissue components, which are known as chromophores, and the others are backscattered to the surface. These backscattered photons carry information about the brain activity. Absorption of NIR light by the scalp, skull and lipids is minimal whereas the chromophores such as oxygenated hemoglobin and deoxygenated hemoglobin are the strong absorbers of NIR light. The concentration levels of these chromophores vary based on activation of the brain. In this work, we present a simple, accurate and low cost NIRS system designed and evaluated its performance applicable in monitoring functional brain activity through the measurement of changes in chromophores.

\section{Methodology and Methods:-}

$O D_{\lambda}=-\log \frac{I_{O}}{I_{i}}=\varepsilon_{\lambda} \cdot C \cdot L_{\lambda}+S_{\lambda}$ On the basis of light emission from the sensor, three types of NIRS devices have been developed by researchers and they are (1) continuous wave (CW) (2) frequency modulated, FM and (3) time resolved (TR). The CW mode NIRS is the simplest and low cost instrument in which a continuous light beam emits from the source that passes through the tissue after which it is detected from a photo-detector. From the readings of the photo-detector, hemoglobin concentrations in the tissue can be determined by applying the modified BeerLambert's law (mBLL) (Rolfe 2000) (Liu et al, 2000) which is expressed in Equation (1). The well known BeerLambert law in optics is used for a transparent, non-scattering medium. Since the biological tissue scatters the light, therefore the Lambert-Beer law is modified to incorporate the scattering effect of the tissue which is known as mBLL.

Where, $O D_{\lambda}$ is the optical density for wavelength $\lambda, I_{i}$ is the incident light, $I_{o}$ is the detected light, $\varepsilon$ - absorption coefficient of a chromophore, $C$ is concentration of the chromophore, $\mathrm{L}_{\lambda}$ is averaged path length and $S$ is the light scattering term. The averaged path length term can be expressed in terms of source to detector gap as given by Equation (2).

$L_{\lambda}=d \cdot D P F_{\lambda}$

Where, ' $\mathrm{d}$ ' is the distance between the light source and the photo-detector and $D P F_{\lambda}$ is the differential path length factor. Due to the light scattering phenomenon in the tissue, a correction factor is considered which a dimensionless DPF is. This factor accounts for the increased optical path length in the scattering medium than that of nonscattering medium, like water. When the optical density is measured at different time instants, then the difference is expressed as below (Eq. 3).

$\Delta O D_{\lambda}=\varepsilon_{\lambda} \cdot[C(t 2)-C(t 1)] \cdot D P F_{\lambda} \cdot d$

or,

$\Delta O D_{\lambda}=\varepsilon_{\lambda} \cdot \Delta C \cdot D P F_{\lambda} \cdot d$

Above equation (Eq. 4) shows that the difference of optical densities is directly proportional to the variation of concentration in time.

When two or more wavelengths are used, a linear system in two equations and two unknowns can be obtained. Selecting two specific wavelengths in NIR range in the interest of two chromophores, the changes of deoxygenated and oxygenated hemoglobin respectively, can be computed from below equations (Eq. 5 and Eq. 6), which are obtained from the linear system.

$$
\begin{array}{r}
\Delta[D H b]=\frac{\varepsilon_{O H b}^{\lambda_{2}} \cdot \Delta O D_{\lambda_{1}}-\varepsilon_{O H b}^{\lambda_{1}} \cdot \Delta O D_{\lambda_{2}}}{\left(\varepsilon_{D H b}^{\lambda_{1}} \cdot \varepsilon_{O H b}^{\lambda_{2}}-\varepsilon_{D H b}^{\lambda_{2}} \cdot \varepsilon_{O H b}^{\lambda_{1}}\right) \cdot L} \\
\Delta[O H b]=\frac{\varepsilon_{D H b}^{\lambda_{2}} \cdot \Delta O D_{\lambda_{1}}-\varepsilon_{D H b}^{\lambda_{1}} \cdot \Delta O D_{\lambda_{2}}}{\left(\varepsilon_{D H b}^{\lambda_{1}} \cdot \varepsilon_{O H b}^{\lambda_{2}}-\varepsilon_{D H b}^{\lambda_{2}} \cdot \varepsilon_{O H b}^{\lambda_{1}}\right) \cdot L}
\end{array}
$$

These parameters $(\Delta[\mathrm{DHb}]$ and $\Delta[\mathrm{OHb}])$ reveal information about the brain activity.

\section{Design of Hardware system}

The NIRS system consists of NIR light sources to pass the NIR light into the tissue, photo-detectors to receive the light emerging from the deeper sections of the tissue, microcontroller and data acquisition system that controls the timing sequence of the sources and electrical signals from the photo-detectors and a storage system which is usually computer connected to the NIRS system for processing and visualization of the data. 


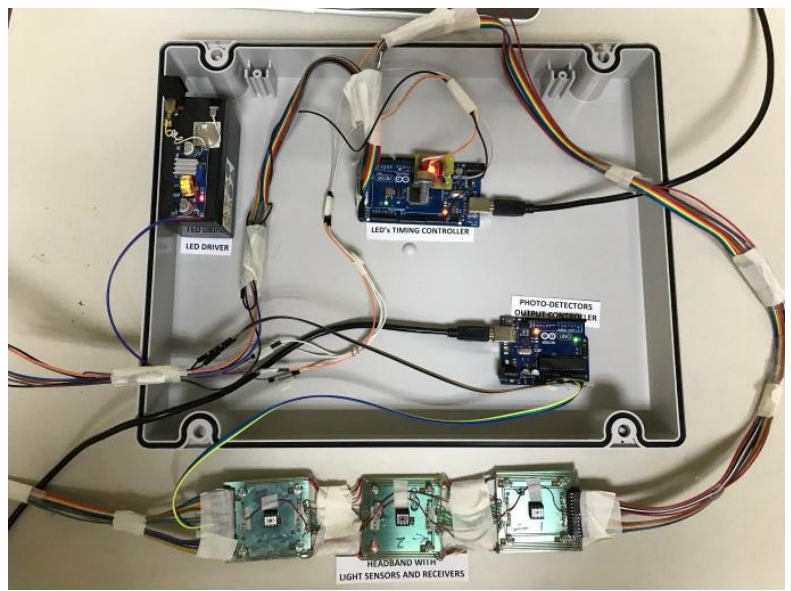

Figure 1:-Top view of the proposed NIRS system with the signal measurement probe.

Figure (1) shows top view of the proposed NIRS system that consists of power supply, LED driver and control circuit, photo-detector output controller and head and carrying light sensors and photo-receivers. In the system two microcontrollers are used (Arduino MEGA, Arduino UNO) for controlling the components and for analog to digital conversion. Optical components are fixed in the forehead band itself and they are connected with the respective microcontrollers through electrical wires rather than using fiber optic cables so that the signal strength and optical power delivery being enhanced. The microcontrollers are interfaced with the optical components and a computer using USB cables. Thus, the output data transmits from photo-detectors to the computer where the acquired data is then analyzed in MATLAB software (MathWorks, Inc.).

\section{Selection of Wavelengths and light sources}

From the fact that the near infrared radiation $(650 \mathrm{~nm}-1100 \mathrm{~nm})$ penetrate through the biological tissue and a narrow optical window of 700-900 nm is optimally suited for NIRS because hemoglobin is the main absorber in this range of wavelength. Figure (2) shows absorption spectra of hemoglobin concentrations in therapeutic optical window. Since the NIRS is based on the differential absorption spectra of the two hemoglobin chromophores, a minimum of two wavelengths need to be selected in the optical window. Referring the absorption spectra, we have selected $760 \mathrm{~nm}$ and $840 \mathrm{~nm}$ wavelengths for the measurement of deoxyhemoglobin and oxyhemoglobin hemoglobin concentrations in the tissue. We used dual wavelength LEDs as light sources L760-840 made from Epitex Company in a 5mm molded package. Each LED emits $760 \mathrm{~nm}$ and $840 \mathrm{~nm}$ wavelengths from the single package as continuous wave.

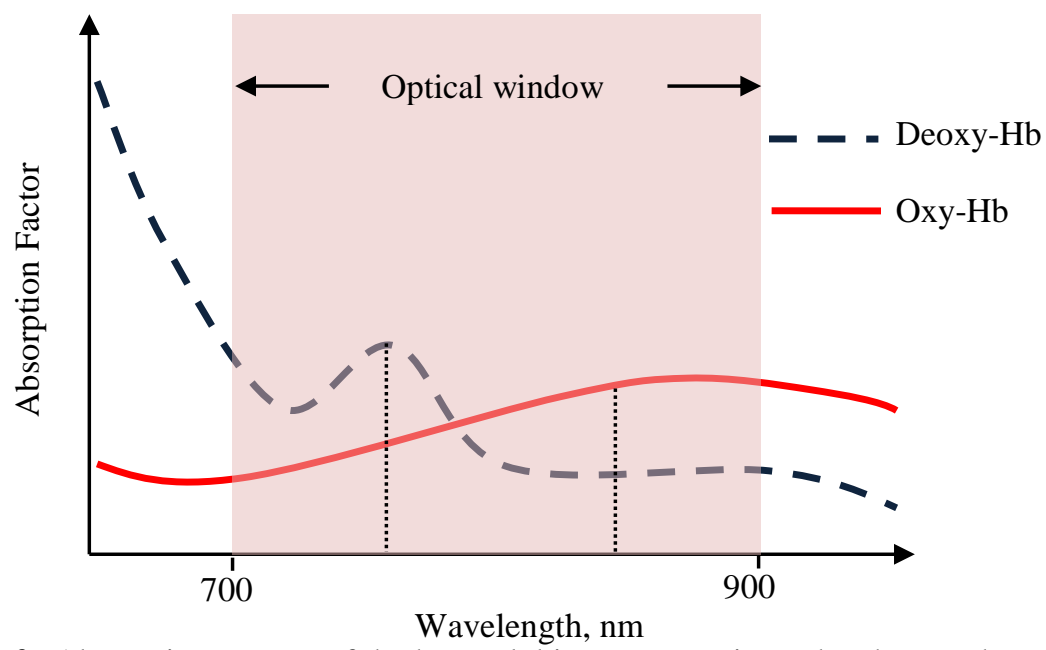

Figure 2:-Absorption spectra of the hemoglobin concentration. The chromophores (deoxy and oxy hemoglobins) have distinct absorption factors nearly at 760 and $840 \mathrm{~nm}$ respectively. 


\subsection{Photodiodes:}

In this project we used OPT101 photodiodes for detecting the optical signal. The photodiode has the highest responsivity for the wavelengths in an optical window of 700-900 $\mathrm{nm}$. The photodiode is in the form of a chip in which photoelectric sensor and pre-amplifier are integrated together.

\subsection{NIRS probe:}

To acquire the signal from the depth of approximately $\sim 15 \mathrm{~mm}$, the LEDs and photodiodes are separated by a distance of $30 \mathrm{~mm}$ (Amog et al., 2011) (Judith et al., 2011). It has been demonstrated by (Chitnis et al, 2016) and (Funane etal 2017) that the modular optodes are well suited for adaptive channel configuration to any individual. Figure (3) shows localization of the sensors and NIR light flow through the head.

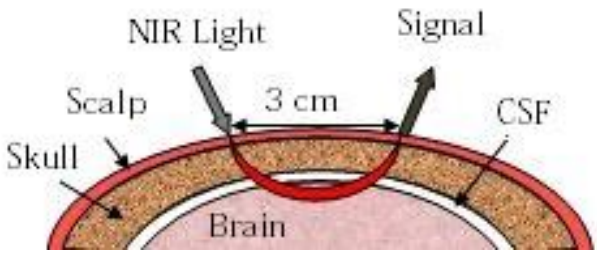

Figure 3:-Separation of LED and photodiode on the surface of the head allowing the NIR light to penetrate deeper into the brain

The probe housing LEDs and photodiodes has been fabricated in rectangular shape that can be placed over the forehead during measurements.

\subsection{Software development}

The LED outputs are pulsed from a microcontroller. A Software program was developed on Arduino platform to control the timing sequence of the 760 and $840 \mathrm{~nm}$ wavelengths from all the LEDs. Each wavelength will be ON for $20 \mathrm{~ms}$ and $5 \mathrm{~ms}$ will remain OFF from every LED. The off-time is given to overcome with cross-talk between the wavelengths. Another Arduino software program was developed to acquire the signal from each photodiode sequentially detected from every wavelength and read the data in digital form in a computer. The data was saved and analyzed in MATLAB software package.

\section{Experimental Results and Discussion}

In order to make assessment, calibration standardization and evaluation of new diagnostic and therapeutic instruments, phantoms are used in the preliminary studies. Phantoms are objects which are made by certain materials mimicking the biological tissues in terms of optical properties, since the source of energy interaction is the light. In the model study of optical tissue interaction, researchers have been used various materials in the form of solids and liquids. Wilson (Wilson, et al 1989) and Mitic (Mitic et al 1994) used milk as fluid phantom simulating the tissue as the milk has similar optical characteristics resembling the tissue in the NIR wavelengths. So, we used non-creamy milk to validate the performance of our NIRS system. In our experiment. A clean and fully transparent glass tank of $20 \times 20 \times 20 \times \mathrm{xcms}$ size was filled with fresh at-free milk diluted in 1:3 by addition of distilled water. An optical absorber, made from $10 \mathrm{~mm}$ diameter black colored plastic rod was immersed in the milk. On the outer surface of the glass tank, the NIRS probe was firmly fixed. The rod was arranged such that it can be moved in the tank during the measurement. From the probe the data was recorded while the rod was manually moving slowly towards and away from the probe surface. Figure (4a). shows the tank, absorber rod and NIRS probe arrangement for conducting the experiment. The data was then saved in the computer and analyzed in MATLAB software. Figure (4b). shows the processed data from one channel of the NIRS probe. In the graph, y axis shows variation of the normalized values of absorbance (optical density, OD) in response to the movement of the rod in the light scattering medium. It can be noted from the graph that the value of absorbance attains maximum when the black rod was $15 \mathrm{~mm}$ away from the probe surface. This shows that the newly developed NIRS system is sensitive to the chromophores also when the spectral wavelengths are taken in consideration. The system therefore will be planned to use in the measurement of changes in hemoglobin parameters during the brain activation. 

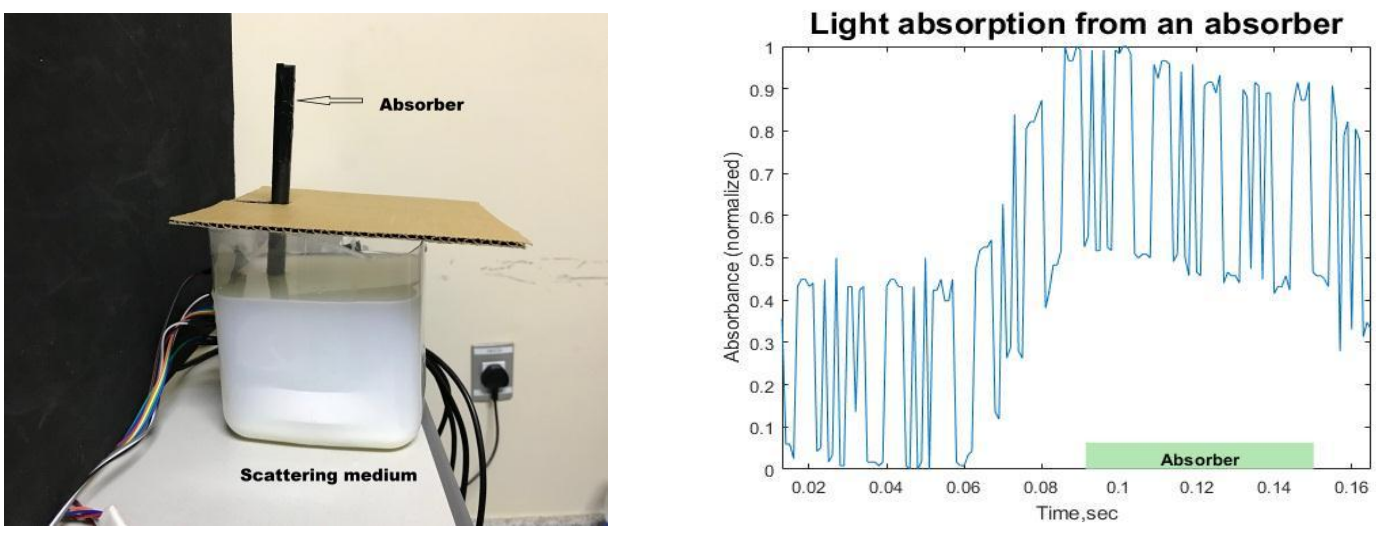

Figure 4:-(a) Experimental setup to measure optical absorption by an absorber in milk medium as tissue phantom and (b) the result.

\section{Conclusion:-}

We have developed and tested a continuous wave NIRS system. Since we used dual wavelength LEDs and transimpedance amplifier photodiodes, the system is portable, safe and low cost. We have used Arduino microcontrollers to interface the hardware with the computer. The multiplexing of LEDs, demultiplexing the photodiode outputs and duty cycle settings of the light sources were done by executing the respective software programs developed in Arduino platform. The result shows that the NIRS system is accurate and sensitive for the measurement of changes in hemoglobin concentration induced due to cortical activation.

\section{References:-}

1. F. F. Jobsis, (1977), Noninvasive, infrared monitoring of cerebral and myocardial oxygen sufficiency and circulatory parameters, Science, vol. 198, no. 4323, pp. 1264-1267.

2. M. Cope and D. T. Delpy (1988). System for long-term measurement of cerebral blood and tissue oxygenation on newborn infants by near infra-red transillumination, Med. Biol. Eng. Comput., vol. 26, no. 3, pp. 289-294.

3. Wilson B, Park Y, Hefetz Y, Patterson M, Madsen S and Jacques S (1989). The potential of time-resolved refelectance measurements for the noninvasive determination of tissue optical properties. Proc. SPIE $106497-$ 106.

4. G. Mitic, J. Kolzer, J. Otto, E. Plies, G. Solkner and W. Zinth, (1994). Time-gated transillumination of biological tissues and tissuelike phantoms, Appl. Opt., 33, 6699-710.

5. P. Rolfe (2000). In Vivo Near Infra-Red Spectroscopy, Annual Reviews in Biomedical Engineering, vol. 2, pp. 315-354.

6. Liu, H., Y. Song, K. L. Worden, X. Jiang, A. Constantinescu and R. P. Mason, (2000). Non invasive investigation of blood oxygenation dynamics of tumors by near-infrared spectroscopy, Applied Optics 39: pp.5231-5243.

7. T. Vaithianathan, I. D. Tullis, N. Everdell, T. Leung, A. Gibson, J. Meek and D. T. Delpy (2004). Design of a portable near infrared system for topographic imaging of the brain in babies. Rev. Sci. Instrum. 75(10), pp. 3276-3283.

8. C. Kim, S. Lee, D. Koh and B. Kim (2011). Development of wireless NIRS system with dynamic removal of motion artifacts. Biomedical Engineering Letters 1(4), pp. 254-259.

9. Amol V. Patil, Javad Safaie, Hamid Abrishami Moghaddam, Fabrice Wallois, and Reinhard Grebe, (2011). Experimental investigation of NIRS spatial sensitivity, Biomed. Opt. Express 2, 1478-1493.

10. Judit Gervain, Jacques Mehler, Janet F. Werker, Charles A. Nelson, Gergely Csibra, Sarah LloydFox, Mohinish Shukla, Richard N. Aslin, (2011). Near-infrared spectroscopy: A report from the McDonnell infant methodology consortium, Developmental Cognitive Neuroscience, Volume 1, Issue 1, 22-46.

11. Chitnis, D., Cooper, R. J., Dempsey, L., Powell, S., Quaggia, S., Highton, D., Elwell, C., Jeremy, C. and Everdell, N. L, (2016). Functional imaging of the human brain using a modular, fibre-less, highdensity diffuse optical tomography system. Biomedical Optics Express, 7, 4275-4288. 
12. Funane, T., Numata, T., Sato, H., Hiraizumi, S., Hasegawa, Y., Kuwabara, H., Hasegawa, K, Kiguchi, M, (2017). Rearrangeable and exchangeable optical module with system-on-chip for wearable functional near-infrared spectroscopy system. Neurophotonics, 5(1), 011007. https://doi.org/10. 1117/1.nph.5.1.011007.

13. Paol Pinti, Ilias Tachtsidis, Antonia Hamilton, Joy Hirsch, Clarisse Aichelburg, Sam Gilbert, and Paul W. Burgess, (2018). The present and future use of functional near-infrared spectroscopy (fNIRS) for cognitive neuroscience, Ann. N. Y. Acad., Special Issue: The Year in Cognitive Neuroscience, 1-25. 